\title{
IYCN practices and nutritional status of young children (0-5 years) in Gujarat: insights from national data sets
}

\author{
Hemangini Gandhi ${ }^{1 *}$, Mousami Shah ${ }^{2}$ \\ ${ }^{\mathbf{1}}$ Assistant Professor, ${ }^{2}$ Research Coordinator, Dept. of Foods and Nutrition, Faculty of Family and Community Sciences, The Maharaja \\ Sayajirao University of Baroda, Vadodara, Gujarat, India
}

*Corresponding Author: Hemangini Gandhi

Email: hgandhi1950@gmail.com

\begin{abstract}
Introduction: People are at the core of sustainable development. Individual wellbeing is essential to sustaining the inter-generational gains in health, productivity and social engagement that underpin the sustainable development agenda. Recently, India stands with a population of 1.37 billion population in which Gujarat has population of 6.27 crores. (World Population Review 2019) ${ }^{11}$. Globally, one in three children under the age of 5 is stunted, wasted or overweight and, in some cases suffers from a combination of two of these forms of malnutrition. India comprises almost 13.1 per cent of child population aged 0-6 years. Manifestations of malnutrition emerge not only from food insecurity but also from poor child care and awareness towards it. The present paper is an attempt to review the status of IYCN practices and child's malnutrition status in Gujarat.

Materials and Methods: Using available NFHS data sheets and other comparable data sets, an attempt is made to understand the magnitude of undernutrition in children below 5 years. The paper is based on the secondary data from various published reports of the government of India.

Main findings: Recently conducted NFHS - 4 provides data on children feeding practice and their Nutritional status. India accounted almost 43 per cent of children underweight against 47 percent in Gujarat. Over the years, feeding Practice like early initiation of breastfeeding within one hr of birth, exclusive breastfeeding and introducing complementary feeding with adequate diet was found to be better in Gujarat State as compared to India. Malnutrition among the children reduced significantly over the time, but still the number of undernourished children is very high in the state.

Conclusion: There is a declining trend in nutritional status of undernutrition in children and improvement in IYCN practices in Gujarat, the concerns still remain for its pace as we need to achieve new targets of Rashtriya Poshan Abhiyan by 2022. The paper reinforces the need for state specific effective delivery and utilization of major government programmes and community participation to achieve targets of Rshtriya Poshan Abhiyan and SDGs at large.
\end{abstract}

Keywords: Undernutrition, Feeding practices Child health, Rastriya Poshan Abhiyan, SDGs

\section{Introduction}

Despite recent achievement in economic progress in India, it has failed to secure a better nutritional status among all children of the country. Growing evidence suggest there exists a socio-economic gradient of childhood malnutrition in India. Nutrition is a fundamental human right and it plays a key role in health. People are at the core of sustainable development. The health status of a Country can largely be gauged by measuring the health of its women and children. Nowadays, we have changed our roles: women are increasingly joining the formal workforce, balancing work responsibilities with their role as primary caregivers, and often with little support from families, employers and societies. Life on our planet has changed: climate change, the loss of biodiversity, and environmental damage now raise concerns over whether we can feed this generation of children sustainably, never mind the generations to come. And we have changed what we eat: we are leaving behind traditional diets and embracing modern diets that are frequently high in sugars and fats, low in essential nutrients. (GOI Rural Health statistics 2006) ${ }^{5}$

Gulnawaz Usnami et, al. (2017) ${ }^{6}$ opined that children of today are tomorrow's citizen, and also the assets for tomorrow's productivity hence it is extremely important to ensure good health for children. Child health plays a vital role in the development of a country. The first six years of life for the children are the most crucial span in their life. At this stage of life, the foundations are laid for mental, physical and social development. The growth of any country is dependent on the availability of healthy human resource. Healthy children ensure for healthy adult who can ensures a sound growth and development of the country. New-borns particularly infant and under-five children are more prone to malnutrition, mortality and other diseases, which can be easily prevented or treated.

\section{Comparison of various national nutritional status of children ( $0-5$ years) in Gujarat state}

The present paper is made to study the time trends on nutritional status and IYCN practices of children ( $0-5$ years) in state of Gujarat. Nutrition is the basic component for healthy life. Adequate nutrition is very essential in the early stages of life. Nutrition helps in the growth and development of body. Indian has the largest food supplementation program in the world. Although country experiences rapid economic growth and easy access to affordable food and food supplementation programmes for children, yet there are nearly half the under-five children are underweight. Another important concern of the paper is status of children in Gujarat state. Despite progress in health sector in India, Gujarat has very high child mortality rates. 
The child mortality rates reflect the threats in child health. Malnutrition is the major cause of mortality among children.

The study is based on the secondary data collected from various government reports such as NFHS reports). Rapid Survey on Children report, Comprehensive National Nutrition Survey reports, etc.

Gujarat state of India has come a long way in improving the health indicators since independence, but progress in reducing maternal mortality has been slow down. One in three children under the age 5 is stunted, wasted or overweight and, in some cases suffers from a combination of two of these forms of malnutrition.

\section{Time trends: Nutritional status of children}

Nutritional status of the children is the most important indicator for the development of the world. In every state the respective governments have made every possible effort to tackle down the prevalence of underweight children. A word once inextricably linked in the public's mind to images of hunger and famine, malnutrition must now be used to describe children with stunting and wasting, but also those suffering from the 'hidden hunger' of deficiencies in essential vitamins and minerals as well as the growing numbers of children and young people who are affected by overweight or obesity.

According to the National Family Health Survey 4 $(2015-16)^{7}, 36$ percent of Indian children are underweight (Figure 1). The health condition of children is worst in India comparatively to other developing countries. Though Country experience higher economic growth but stands far behind in terms of underweight children in the world comparatively to other developing countries.

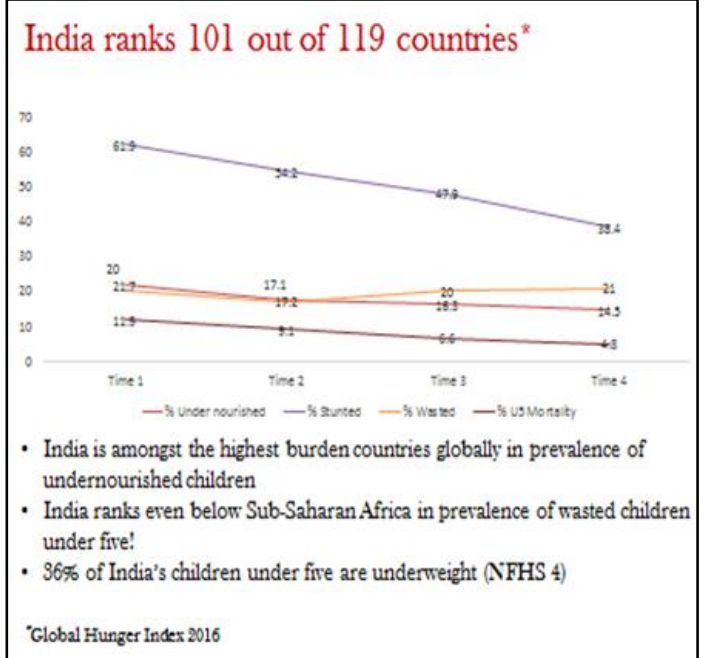

Now, time trends in comparison of demographic and health status of children of India and Gujarat it was found that shows Gujarat is doing somehow better than the national average for most demographic and health indicators. No much difference is found in fertility rate of Gujarat and India. Its infant mortality rate (IMR) is 34 versus the national rate of (41), and the Under 5 child mortality rate (U5MR) is 43 vs 50 respectively. (Table 1)

According to former Vice President of India, Hamid Ansari: "it seems the state you are born in determines how long you live". Indicators used for measuring children malnutrition are based on weight-for-age, height-for-age and weight-for-height. The weight-for-height index measures body weight in relation to body height, case of acute malnutrition (Wasting). Height-for-age index measures growth retardation (Stunting) among children, case of chronic malnutrition. Weight-for-age index reflects both the case of chronic and acute (Undernutrition) and Anemia among children. V.K Paul (2018) ${ }^{10}$ Key issues in childhood undernutrition are shown in Fig. 2.

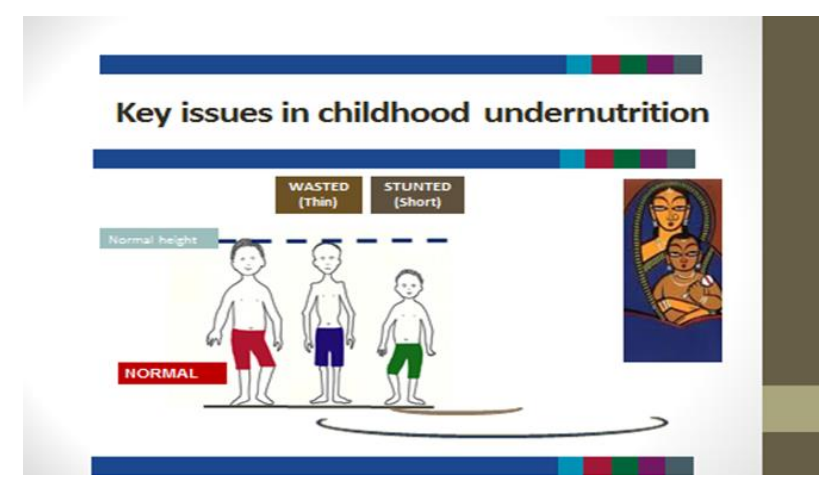

Fig. 2:

Fig. 3 represents the percentage change of nutritional status in children (0-5 years) in Gujarat state over the time. In the (RSoc 2014-15) ${ }^{9}$ survey 43 percent of children are stunted in the state which gradually got down to 39 percent in CNNS 2018-19 survey. Change in percentage found to be only 4 percent which seems to be very less. According to National Family Health Survey 4 which is carried out in 2015-16 showed that 26 percent of the children are found to be which got reduced to 17 percent in (Comprehensive National Nutrition Survey $2017-18)^{2}$. No much difference is found in the percentage of children who are wasted and underweight in Gujarat state from 2014-2019. Trends from RSoC to CNNS show an overall no significant declined in stunted, wasted and underweight children in the country.

Fig. 1:

Table 1: Demographic and Health indicators of India and Gujarat (NFHS-4 2015-16)

\begin{tabular}{|l|c|c|}
\hline \multicolumn{1}{|c|}{ Indicator } & India & Gujarat \\
\hline Population (World population 2019) & 1.37 billion & 63.8 million \\
\hline $\begin{array}{l}\text { Sex ratio at birth for children born in the last five years } \\
\text { (females per 1,000 males) }\end{array}$ & 919 & 907 \\
\hline Total fertility rate (children per woman) & 2.2 & 2.0 \\
\hline
\end{tabular}




\begin{tabular}{|l|c|c|}
\hline Children under age 5 years whose birth was registered (\%) & 79.7 & 95.8 \\
\hline Infant Mortality Rate & 41 & 34 \\
\hline Under 5 mortality rate & 50 & 43 \\
\hline
\end{tabular}

Table 2 represents Haemoglobin levels defining Anemia among children (6-59 months) (WHO-2018) ${ }^{\mathbf{1 2}}$

\begin{tabular}{|c|c|c|c|c|c|}
\hline Age and Sex & \multicolumn{5}{|c|}{ Haemoglobin levels gm/dl } \\
\hline & Non Anemic & Anemic & Moderate Anemia & Mild Anemia & Severe Anemia \\
\hline Children 6-59 months & 11.0 or more & $<11.0$ & $10-9.99$ & $7-9.99$ & $<7.0$ \\
\hline
\end{tabular}

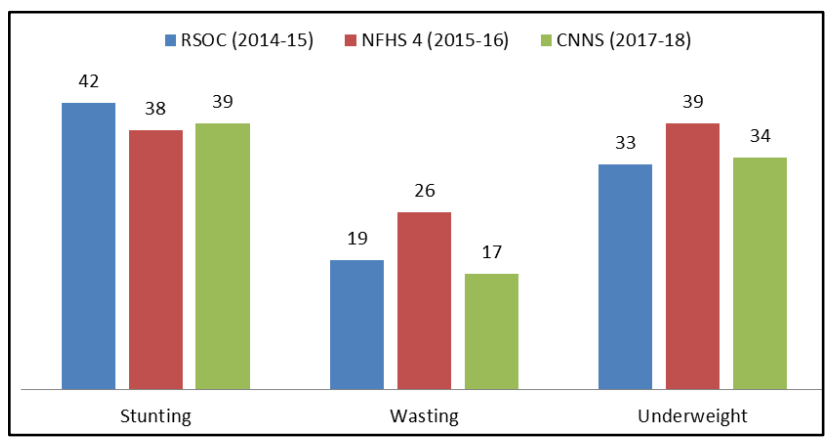

Source: RSOC (2014-15), NFHS 4 (2015-16) and CNNS (2017-18)

Fig 3: Trends in Nutritional status of Children (05 years) in Gujarat

Infant and young child feeding practices

Optimal feeding practices include initiation of breastfeeding within an hour of birth, exclusive breastfeeding for the first six months, continued breastfeeding for two years or beyond, along with adequate and appropriate complementary feeding beginning after six months. The World Health Assembly (WHA) set targets on nutrition including increasing global rates of exclusive breastfeeding from $38 \%$ in 2012 to $50 \%$ 2025. India is committed to achieve these WHA (WHA, 2012) and Sustainable Development targets. (Gupta and Thakur 2018) ${ }^{1}$

\section{Importance of Optimal Feeding Practices}

Patel and Bansal et, al. (2015) ${ }^{3}$ also opined that Optimal feeding practices are very essential for babies not because they are vulnerable but because it is the critical time when brain grows faster and maximum e.g. $80-85 \%$ brain growth takes place during first two years. Malnutrition contributes to about $60 \%$ of under-five mortality worldwide annually and over two-thirds of these are due to inappropriate feeding practices. The World Health Organization (WHO-2018)12 recommends exclusive breastfeeding for the first six months of life and the addition of complementary feeds from six months onwards, with continued breastfeeds till at least two years of age. Apart from exclusive breastfeeding initially, time of introduction, content, and consistency of complementary feeds are critical for early nutrition. The early introduction of complementary feeds before the age of six months can lead to displacement of breast milk and increased risk of infections, besides the babies being physiologically immature. Similarly, inadequate and inappropriate complementary feeding with unhygienic practices leads to recurrent and persistent infections and malnutrition which is followed by growth retardation, immunodeficiency, and eventually fatal outcomes. This is a concern for Indian scenario, where previous studies have suggested inability to maintain exclusive breastfeeding and late introduction of complementary feed. Breastfeeding though is a natural act; it is a behavior that needs to be learned. Mothers and other caregivers need active assistance for optimum breastfeeding practices.

\section{Early Breastfeeding Within one Hour of Birth}

This and skin-to-skin contact provide immense benefits to both, the mother and the child. It can contribute a great deal to reduce neonatal infections and impacts infant mortality. It also helps in emotional bonding and maintenance of baby's temperature. (Gupta and Thakur 2018) ${ }^{1}$

\section{Exclusive Breastfeeding (0-6 months)}

An analysis in Lancet 2016 (Victora et al., 2016) concluded that scaling up breastfeeding (exclusive for first six months and continued for 12 months) to nearly universal levels could prevent nearly $50 \%$ of diarrhea episodes and $1 / 3$ rd of respiratory infections. It could save lives of more than 820,000 children worldwide. Breastfed children perform better on intelligence tests and are less likely to be obese or overweight and they are less prone to diabetes later in life. (Gupta and Thakur 2018) ${ }^{1}$

\section{Complementary Feeding}

Patel and Bansal et, al. $(2015)^{3}$ also opined that exclusive breastfeeding and appropriate complementary feeding have the potential to simultaneously reduce the risk of wasting, stunting as well as overweight/obesity or diet-related NCDs (WHO, 2016). Dietary diversity in children in 6-23 months age group is a proxy marker for micronutrient density (NFHS-4).

\section{Infant and Young Child Feeding Practices: Time Trends and Current Status}

Dr. JP Dadhich $(2019)^{4}$ said that in India 3 out of 5 newborns don't get to begin breastfeeding within an hour and also 1 out of 2 women is not able to exclusively breastfeed her baby. 


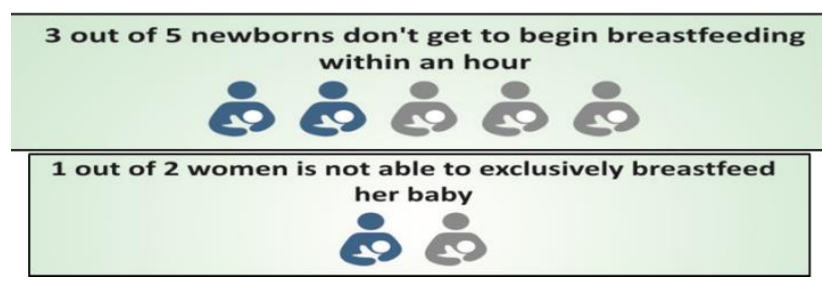

In Gujarat state as shown in Fig 4 between NFHS-3 and 4, there has been an increase in early initiation of breastfeeding from 27.1 to $50 \%$. Data from NFHS- 4 indicates that during 0-6 months, $47.8 \%$ women exclusively breastfeed their infants (improvement of $8 \%$ /year as compared to NFHS-3). Regarding Complementary feeding it is found that according to NFHS-4 data, only $49.4 \%$ of infants get complementary feeds at 6-8 months (Fig. 4). Between NFHS-3 and 4 , there has been a $5 \%$ decline in infants receiving complementary feeds in 6-8 months. Further, According to NFHS 4 data only 5.2 percent of children age (6-23 months) is receiving an adequate diet which means a diet with proper quality, quantity and frequency.

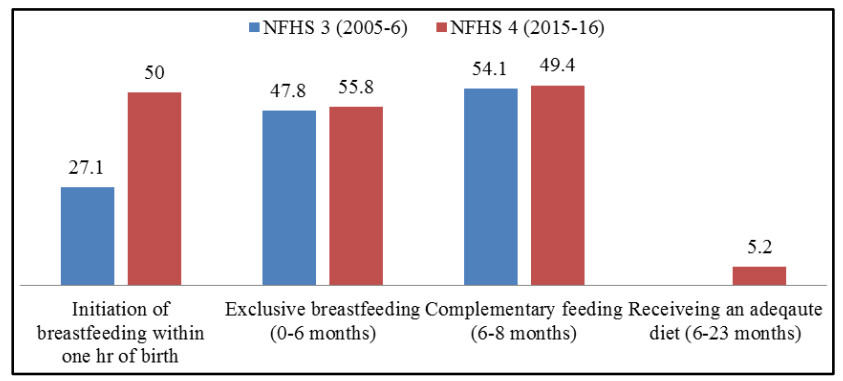

Source: National Family Health Survey 2005-6 and 201516)

Fig. 4: Status of Infant and Young Child Feeding in Gujarat
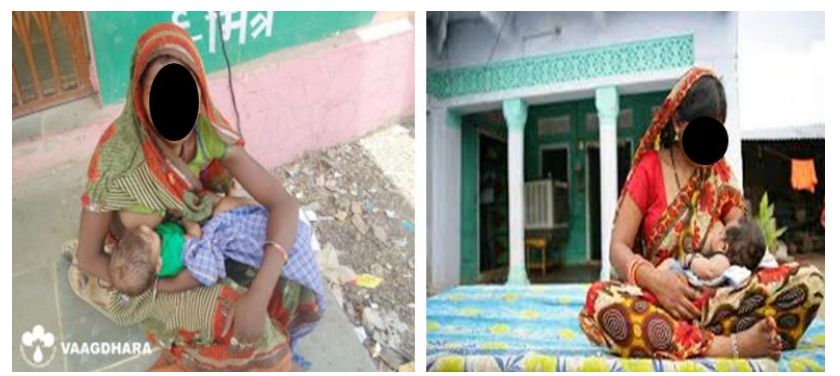

Fig. 5:

\section{Status of anemia among children (0-5 years)}

Vrajlal in $(2016)^{8}$ opined that Anemia prevalence in young children continues to remain over $70 \%$ in most parts of India and Asia despite a policy being in place and a program that has been initiated for a long time. The irreparable damage that anemia in childhood can cause particularly to the development of a young child on one hand and the knowledge and mechanism available for its control on the other, makes this silent morbidity completely unacceptable in modern times where we strive for millennium development Goal 4.

\section{Nutritional deficiency anemia in children}

The term 'nutritional anemia' encompasses all pathological conditions in which the blood hemoglobin concentration drops to an abnormally low level, due to a deficiency in one or several nutrients. The ain nutrients involved in the synthesis of hemoglobin are iron, folic acid, and vitamin B12. In public health terms, iron deficiency is by far the first cause of nutritional anemia worldwide. An abnormally low hemoglobin level due to pathological condition(s) is defined as anemia. Causes of anemia include chronic infections, particularly malaria, hereditary hemoglobinopathies, and folic acid deficiency. (Vrajlal 2016) ${ }^{8}$

Anemia is most often a hidden deficiency, with a few overt symptoms. Policy makers often fail to recognize the massive economic costs, service providers often fail to recognize the significant health consequences, and societies are too often ignorant of anemia's capability to cause permanent cognitive defects, denying children their right to full mental and emotional development, before they ever reach a classroom. In infants and children, a large amount of iron is required for growth (i.e., substantially more iron must be absorbed than is lost from the body). (S.K Ujjwal 2016) ${ }^{13}$

The three main reasons for Iron Deficiency Anemia in children are:

1. Poor bioavailability of iron consumed,

2. Insufficient intake of iron as per the requirement

3. Increased requirement during the rapid growth stage of infancy and early childhood, between six and twentythree months.
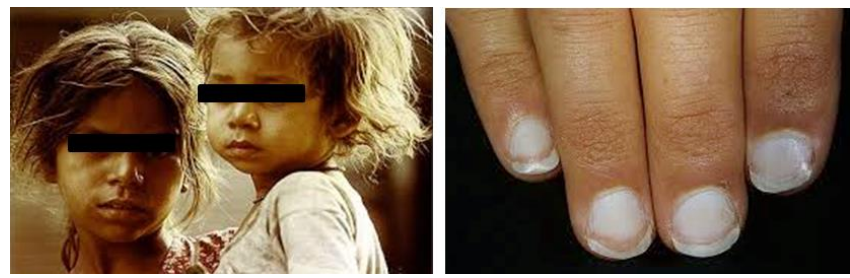

Fig. 6:

Magnitude of Anemia in Gujarat AMONG CHILDREN (6-59 months)

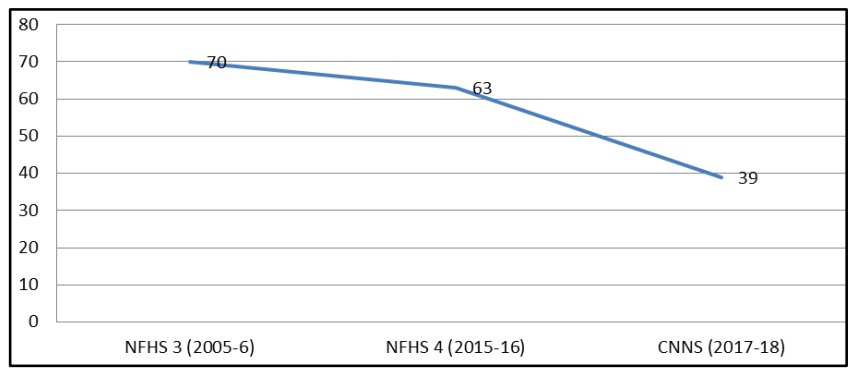

Anemia has been a big problem in Gujarat and the National Family Health Survey (NFHS) 4 data showed the prevalence of anemia among children less than five years of age to be around $63 \%$. When we look at the data for anemia prevalence among children under five years of age, it jumps 
to $70 \%$ and this survey done ten years prior to the NFHS 4 survey, which was done in 2005 - 2006. Only 7 percent reduction has been observed in 10 years of period. However, it is encouraging to know that 24 percent decline is found in 2 years of span in the status of anemia of children (6-59 months).

\section{Conclusion}

It can be concluded that there is a declining trend in prevalence of undernutrition and Anemia in children and improvement in IYCN practices status in Gujarat. The concerns still remain for its pace as we need to achieve new targets of Rashtriya Poshan Abhiyan by 2022. The paper reinforces the need for state specific effective delivery and utilisation of services of major government programmes and community participation to achieve targets of Rashtriya Poshan Abhiyan and SDGs at large.

\section{Source of funding}

None.

\section{Conflict of interest}

None.

\section{References}

1. Gupta A and Thakur N. Infant and Young Feeding Practices in India: Current Status and Progress Towards SDG Targets Review article Research Gate. 2018
2. Comprehensive National Nutrition Survey. Ministry of Health and Family welfare/(2017018)

3. Patel DV, and Bansal. Breastfeeding Practices, Demographic Variables, and Their Association with Morbidities in Children Advances in Preventive Medicine Volume 2015, Article ID 892825

4. Dadich JP (Paediatrics) (2019), FNNF National Coordinator and Technical Director, Breastfeeding Promotion Network of India (BPNI) World Breastfeeding week

5. Government of India. Bulletin on rural health statistics in India. Ministry of health and family welfare, New Delhi. 2006.

6. Usmani G, and Nighat. Ahmad Department of Economics, Aligarh Muslim University, India Journal of Perioperative \& Critical Intensive Care Nursing: Health Status of Children in India, 2015

7. National Family Health Survey. International Institute of Population Sciences, Mumbai, India. (2005-6) \& (2015-16)

8. Kotecha PV, Medical College, Baroda April. Nutritional Anemia in Young Children with Focus on Asia and India, Indian Journal of Community Medicine. 2016

9. Rapid Survey on Children. Ministry of women and child development. Government of India. (2013-2014)

10. Paul VK. Presentation on Health and Nutrition of children(2018)

11. World population review 2019

12. World Health Organization. Report of Children status(2018).

13. UKE ppt, Ujjwal S.K Consequences of Anemia in Maharashtra Ministry of Women and Child Development(2016).

How to cite: Gandhi H, Shah M. IYCN practices and nutritional status of young children ( $0-5$ years) in Gujarat: insights from national data sets. IP J Nutr Metab Health Sci 2020;3(1):1-5. 